\begin{abstract}
¿Cómo citar este artículo?
Hernández-Betancur, J. E., Montoya Restrepo, I. A., y Montoya Restrepo, L. A. (septiembre-diciembre, 2020). Estrategias deliberadas y emergentes y su relación con el proceso de duelo psicológico: Resultados de un taller en gestión estratégica. Revista Virtual Universidad Católica del Norte, (61), 267-282. https://www.doi.org/10.35575/rvucn.n61a16
\end{abstract}

\title{
Estrategias deliberadas y emergentes y su relación con el proceso de duelo psicológico: Resultados de un taller en gestión estratégica'
} Deliberate and emerging strategies and their relation to the process of psychological mourning: Results of a workshop in strategic management

\section{Juan Esteban Hernández-Betancur}

Candidato a Doctor en Ingeniería - Industria y Organizaciones

Facultad de Minas, Universidad Nacional de Colombia Medellín, Colombia

jehernanb@unal.edu.co

Orcid: https://orcid.org/0000-0002-4060-6803

CVLAC: https://scienti.minciencias.gov.co/cvlac/visua-

lizador/generarCurriculoCv.do?cod_rh=0001472214

\section{Iván Alonso Montoya Restrepo}

Doctor en Ciencias Económicas

Facultad de Ciencias Agrarias, Universidad Nacional

de Colombia

Medellín, Colombia

iamontoyar@unal.edu.co

Orcid: https://orcid.org/0000-0003-0959-3466

CvLAC: http://scienti.colciencias.gov.co:8081/cvlac/vi-

sualizador/generarCurriculoCv.do?cod_rh $=0000191370$

Recibido: 23 de mayo de 2020 Evaluado: 31 de julio de 2020

Tipo de artículo: Investigación Científica y Tecnológica

\section{Luz Alexandra Montoya Restrepo}

Doctora en Ciencias Económicas

Facultad de Minas, Universidad Nacional de Colombia Medellín, Colombia

lamontoyar@unal.edu.co

Orcid: https://orcid.org/0000-0002-4896-1615

CVLAC: http://scienti.colciencias.gov.co:8081/cvlac/vi-

sualizador/generarCurriculoCv.do?cod_rh $=0000191264$

1 Documento resultado de la tesis de maestría de Juan Esteban Hernández Betancur, titulada: “Contribución a la comprensión del momento de la decisión estratégica. Formulación de un taller de estrategia orientado al momento de la decisión" en la Universidad Nacional de Colombia (Medellín), con avances presentados en International Educational Technology Conference - IETC2015, Estambul, Turquía, del 27 al 29 de marzo del 2015. 


\section{| Resumen}

Los agentes generan vínculos con la estrategia deliberada y la posibilidad de surgimiento de la estrategia emergente; proceso que puede ser explicado por el duelo psicológico. El documento tuvo como propósito identificar la relación entre el ciclo de las estrategias deliberadas y las estrategias emergentes, y el proceso de duelo psicológico. Se desarrolló un taller de estrategia basado en el "juego de la cerveza", con posterior aplicación de una encuesta que buscaba identificar la percepción de los participantes. Se estableció una relación teórica del proceso de duelo psicológico y el ciclo entre las estrategias deliberadas y emergentes, a partir de la ruptura del vínculo del agente con el plan y la sensación de las etapas del proceso de duelo. Además, se identificó la declaración (por parte de los participantes) de la existencia de un vínculo entre el agente y el plan, y la existencia de las etapas del duelo psicológico dentro de la ruptura de este. Lo anterior, permite conocer los estados por los que deben pasar los agentes para darle continuidad a su viabilidad dentro de los sistemas, para tomar decisiones por medio de estrategias emergentes.

Palabras clave: Ciclo; Duelo; Estrategias deliberadas; Estrategias emergentes; Proceso de duelo.

\section{Abstract}

The agents generate links with the deliberate strategy and the possibility of the emergence of the emergent strategy; a process that can be explained by psychological grief. The document aimed to identify the relationship between the cycle of deliberate and emergent strategies and the process of psychological grief. A strategy workshop based on the "beer game" was developed, with subsequent application of a survey that sought to identify the perception of the participants. A theoretical relationship was established between the psychological mourning process and the cycle between deliberate and emergent strategies, based on the rupture of the agent's link with the plan and the sensation of the stages of the mourning process. In addition, the declaration (by the participants) of the existence of a link between the agent and the plan, and the existence of the stages of psychological mourning within the rupture of the plan were identified. This allows us to know the states through which the agents must pass in order to give continuity to their viability within the systems, to make decisions by means of emergent strategies.

Keywords: Cycle; Mourn; Deliberate strategies; Emergent strategies; Mourning process.

JEL: L1 Market Structure, Firm Strategy, and Market Performance; L2 Firm Objectives, Organization, and Behavior 


\section{Introducción}

La generación conceptual de las estrategias deliberadas y emergentes que hacen Mintzberg (1978, 1987), Mintzberg \& Jorgensen (1987), Mintzberg \& McHugh (1985), y Mintzberg \& Waters (1985), permite un importante avance en el entendimiento del concepto de estrategia y su proceso de formación. Mintzberg y sus coautores explican el comportamiento y características de estas estrategias, a partir de dos tipos de estrategias básicas: las pretendidas y las realizadas; complementan sus contribuciones al vislumbrar un posible ciclo entre las estrategias deliberadas y emergentes, por la vía del aprendizaje en las organizaciones. Montoya (2010), y Montoya y Montoya (2013) desarrollan la idea de ciclo, mencionada por Mintzberg (1987), lo que da lugar a una conexión intertemporal entre las estrategias pretendidas y realizadas.

En el proceso de generación de estrategias deliberadas, el agente constituye un vínculo con su plan, propio de las estrategias deliberadas, que se encuentra en riesgo de romperse cuando es sometido a interacción con el ambiente, por apariciones de situaciones truncadoras que lo deconstruyen, impidiendo su ejecución exitosa, desde el alcance de los objetivos. La ruptura de ese vínculo invita a analizar sobre la posibilidad de surgimiento de un proceso de duelo psicológico, presunción basada en las concepciones hechas por de Freud (1917, como se citó en Zaragoza, 2007), enlazadas a que duelo es la respuesta natural a la ruptura del vínculo, y que puede estar asociada a representaciones como los ideales (J. Hernández et al., 2015, 2017, 2019).

El análisis puntual del proceso de duelo se hace teniendo en cuenta las aportaciones hechas por Kübler-Ross (1993), quien realiza sus estudios con base en la aceptación de la muerte, y concluye que se presentan cinco etapas: negación, ira, negociación, depresión y aceptación de la pérdida o ruptura del vínculo.

En este orden de ideas, este artículo busca hacer una evaluación, haciendo uso del reconocido "juego de la cerveza”, de las sensaciones que genera la deconstrucción de la estrategia deliberada; esto es, la imposibilidad de continuar ejecutando un plan y una proyección prevista por un agente, ya que eventos del mundo real paralizan su implementación, generan consecuencias sobre el agente asociadas al duelo psicológico y requieren surgimiento de estrategias emergentes (J. Hernández et al., 2015, 2017, 2019).

A continuación, se enuncia una noción adaptativa de la estrategia, se exponen algunas definiciones del duelo y las etapas del proceso, propuestas por Kübler-Ross (1993), y cómo este se relaciona con la ruptura del vínculo entre agente y la estrategia deliberada. En la segunda parte, se realiza una propuesta de taller basado en el "juego de la cerveza", para validar las etapas del proceso del duelo al momento de la pérdida del vínculo entre el agente y la estrategia deliberada. El tercer apartado se encarga de exponer los resultados de la aplicación del taller; en el cuarto se presentan las discusiones; y, finalmente, en la quinta parte se exponen las conclusiones.

Noción adaptativa de la estrategia. El enfoque de la formación de la estrategia como un proceso adaptativo, a diferencia del racional, destaca las actividades que realizan los agentes para entrar en armonía adaptativa con el entorno y con ellos mismos; invita a una evaluación de largo plazo y se centra en alcanzar algún objetivo de eficiencia económica y soluciones de situaciones problema adaptativas, por medio de la dominación del "entramado de producción". Este último es considerado la estructura social de la organización: instituciones, combinaciones y rutinas, coaliciones políticas entre grupos de interés (J. Hernández, 2015; Montoya, 2010). 
En este enfoque adaptativo, se entiende que los agentes se encuentran caracterizados por la racionalidad limitada, al momento de tomar decisiones, soportado en las aportaciones de autores como March \& Simon (1958) y Simon (1955), donde los agentes están inmersos en el problema de la búsqueda de una solución satisfactoria, más que una óptima (J. Hernández, 2015; Montoya, 2010).

De hecho, entre el enfoque adaptativo, desde la escuela de recursos y capacidades, y el enfoque racional, con la escuela de posicionamiento, se han creado los límites opuestos de la teorías de la formación de la estrategia y han alejado a los investigadores de una concepción de la estrategia ( Londoño-Patiño y Acevedo-Álvarez, 2018; Montoya, 2010; Montoya y Montoya, 2013), que permita, según Mintzberg (1978), vislumbrarla como un patrón en un flujo de decisiones (J. Hernández, 2015).

J. Hernández (2015), señala que Mintzberg realiza una crítica respecto a la concepción de la estrategia solo como algo explícito (Mintzberg, 1978, 1987; Mintzberg \& Jorgensen, 1987; Mintzberg \& McHugh, 1985; Mintzberg \& Waters, 1985); que dicha noción es incompleta para la organización y no operacional para el investigador. Además, que el investigador es forzado a estudiar la formación de la estrategia como algo perceptual, lo que resulta en generalizaciones normativas abstractas (Mintzberg, 1978). De hecho, desconoce la adaptación continua al entorno por parte de las organizaciones, con una actitud activa direccionada por la estrategia (Hax \& Majluf, 1988). Igualmente, se debe reconocer el ecosistema de la estrategia como algo complejo, que va más allá de la visión mecánica de la organización (MacLean \& Maclntosh, 2015).

Montoya (2010) resalta que la invitación de Mintzberg, con la instauración de la noción adaptativa de la estrategia, es estudiar este problema desde una perspectiva evolutiva, que incluya mecanismos de retroalimentación, los cuales contribuyan al aprendizaje organizacional y vinculen percepciones de futuro que permitan la anticipación a situaciones; lo anterior, entendido desde las estrategias deliberadas y emergentes.

Estrategias deliberadas y emergentes. Mintzberg (1987), identifica que algunos planes pueden ser intencionados y pueden ser realizados o no; de ahí parte el concepto de estrategias pretendidas, las cuales surgen de una concepción anticipada de la situación para la consecución de un objetivo, y las estrategias que simplemente son realizadas.

Desde las estrategias realizadas, Mintzberg (1987) identifica que existen estrategias que fueron realizadas y pretendidas; esto concluye en un esfuerzo deliberado por parte del agente, lo que lleva a la consecución de una estrategia deliberada. También, existen las estrategias no realizadas, que básicamente son las estrategias que fueron pretendidas pero que no llegaron a ser ejecutadas. Finalmente, existen estrategias que fueron realizadas, pero no hubo intención anterior por parte del agente para llegar a ese resultado; esto indica que es una estrategia emergente, como producto del seguimiento de patrones y una ausencia de pretensión.

Para que las estrategias sean definidas como deliberadas deben tener una formación exacta de la estrategia realizada a partir de la pretendida, y cumplir satisfactoriamente con tres condiciones (J. Hernández, 2015; Mintzberg \& Waters, 1985; Montoya, 2010):

a. Es necesaria una intención, que no permite ambigüedades, por parte de la organización, con un nivel de detalle concreto que no muestra ninguna duda antes de que sea generada la acción.

b. Las intenciones deben ser comunes para todos los miembros de la organización, ya que se concibe a esta como una acción colectiva.

c. Las acciones del colectivo no pudieron verse afectadas por ninguna fuerza externa y como consecuencia la estrategia pretendida llegó a ser realizada, ejecutándose a cabalidad como se había concebido. Esto da cuenta de un ambiente que es totalmente predecible, dócil y maleable 
No quiere decir que las estrategias emergentes se conviertan en sinónimo de falta de administración; por el contrario, surgen del incrementalismo lógico (Quinn, 1978) de los procesos de planeación, que se transforman en aprendizajes organizacionales, los cuales permiten responder a situaciones no programadas con un patrón viable; concepto similar al de la discontinuidad (Ghezzi, 2013) y la deconstrucción (J. Hernández et al., 2019). Por ende no se puede negar la necesidad de las estrategias deliberadas dentro de la organización, ya que pretenden una dirección acertada hacia los objetivos y el surgimiento de estrategias emergentes (Hax \& Majluf, 1988; J. Hernández et al., 2019; Mintzberg \& McHugh, 1985; Montoya y Montoya, 2013).

Complementando las nociones anteriores, Mintzberg hace énfasis en la formación de la estrategia estudiada como un ciclo entre las estrategias deliberadas y las estrategias emergentes (Montoya, 2010).

Ciclo entre estrategias deliberadas y emergentes. Trascendiendo el esboce que hace Mintzberg (1987) de la idea de ciclo, Montoya $(2010)$ y Montoya y Montoya $(2009,2013)$ hacen un esfuerzo considerable por explicar el funcionamiento del ciclo entre las estrategias deliberadas y emergentes. Por medio de la gestión de aprendizajes ex ante y ex post se llega a una evolución del agente, expuesta desde las concepción de las estrategias deliberadas y emergentes (Montoya, 2010).

Esta concepción cíclica, desde lo evolutivo, queda en evidencia a partir de evaluar antes de iniciar una vuelta del ciclo y luego de ser finalizada, para realizar una comparación de la percepción de la situación en los dos momentos, donde es posible percibir el potencial enriquecimiento cognitivo, por parte del agente, a través de la experiencia (Montoya y Montoya, 2013). Esto le permite al agente mantenerse viable en el sistema para tomar decisiones, dado que hace frente a las novedades, que surgen de su relación sistémica, sin la necesidad de tener previa configuración (Montoya y Montoya, 2013).

Es decir, como queda plasmado en J. Hernández (2015) y J. Hernández et al. (2015), teniendo en cuenta las aportaciones de Montoya y Montoya $(2009,2013)$ y Montoya $(2010)$, el ciclo entre las estrategias deliberadas y estrategias emergentes permite una evolución del agente por medio del reciclaje de aprendizajes, esto combinado con la elaboración de posibilidades. Con esta evolución le permite al agente mantenerse viable dentro del nicho actual o por lo menos tener la posibilidad de instalarse en otro nicho que si le permita ser viable.

El proceso de duelo y el proceso de formación de la estrategia. Para darle sentido a la investigación de la estrategia y la decisión desde el aspecto de las emociones que influyen en los agentes (A. Hernández, 2006; Kariman et al., 2014; Paulus, 2005; Saavedra, 2006), se tiene en cuenta la perspectiva del proceso de duelo, el cual describe las sensaciones de los agentes ante la ruptura de un vínculo (J. Hernández, 2015).

Haciendo un recorrido por las definiciones del duelo, se puede identificar que este se asocia con dolor, lástima, aflicción o sentimiento (Real Academia Española, 2014). Además, se puede considerar como una respuesta natural a la pérdida de algo significativo (Americam Psychiatric Association, 2014).

John Bowlby (1993), el autor pionero y más destacado en la Teoría Afectiva (Fonseca, 2010), presenta una comparación del duelo con una inflamación, es decir una respuesta a una aflicción. Lo que le permite al autor hacer la siguiente definición de duelo: “una serie bastante amplia de procesos psicológicos que se ponen en marcha debido a la pérdida de una persona amada, cualquiera sea su resultado" (Bowlby, 1993, p. 40).

Además, Bowlby (1993) retoma a Freud (1960) con el concepto "Trabajo del Duelo", que, de manera textual, es:

El esfuerzo que hace el individuo para aceptar un hecho ocurrido en el mundo exterior (la pérdida del objeto catectizado) y para llevar a cabo cambios correspondientes en el mundo interior (retiro de la libido del objeto perdido, identificación con el objeto perdido). ( p. 41) 
En tal sentido, el duelo se convierte en una serie de sentimientos que surgen como respuesta natural a la pérdida, donde el agente hace un trabajo de duelo, en el cual elabora una búsqueda de la aceptación del hecho ocurrido (Sánchez, 2020), que en gran medida es evidente a partir de la culpa (Feigelman \& Cerel, 2020). Además, teniendo en cuenta a Freud (1917, como se citó en Zaragoza, 2007), el proceso de duelo puede estar asociado a abstracciones tan significativas como la patria, la libertad y los ideales.

Proceso del duelo. Kübler-Ross (1993), en sus estudios sobre la muerte, determinó cinco fases que experimenta una persona que está próxima a morir o que está dentro de un proceso paliativo; además, también aplicables en rupturas de un vínculo (ver tabla 1).

\section{Tabla 1}

Etapas del proceso de duelo

\begin{tabular}{|c|c|}
\hline Etapa & Descripción \\
\hline 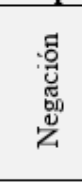 & $\begin{array}{l}\text { - Cuestionamiento y defensa provisional de la realidad. } \\
\text { - Imposibilidad de afectación por la patología que los conduzca al final de la vida. } \\
\text { - Consulta de otras opiniones médicas en búsqueda de un diagnóstico positivo. } \\
\text { - En pocos casos se llega a mantenerse la etapa de negación hasta el final. } \\
\text { - Desvirtuación y encubrimiento de la gravedad de la situación. }\end{array}$ \\
\hline ๘ & $\begin{array}{l}\text { - Transición de la realidad a compresión y aceptación de la realidad de manera parcial. } \\
\text { - Búsqueda de la respuesta a la pregunta de ¿Por qué es él elegido a estar en esa } \\
\text { situación? } \\
\text { - El agente expresa ira ante su entorno como consecuencia de la situación actual. } \\
\text { - Dolor, lágrimas, culpabilidad, vergüenza y evita el contacto con personas cercanas. } \\
\text { - Enojo por la ruptura de las actividades cotidianas y la destrucción de la concepción } \\
\text { de futuro. }\end{array}$ \\
\hline 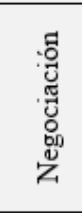 & $\begin{array}{l}\text { - Sabe, por experiencias pasadas, que el buen comportamiento puede obtener } \\
\text { beneficios. } \\
\text { - La negociación surge en todos los ámbitos de las personas, en particular se puede } \\
\text { observar en el espiritual, en donde se entra en negociación, con un ser superior, la } \\
\text { aceptación de la enfermedad a cambio de que sea más llevadera y menos tortuosa } \\
\text { hasta el final. }\end{array}$ \\
\hline 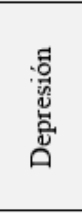 & $\begin{array}{l}\text { - Surge ante la imposibilidad de mantener una actitud positiva, por un deterioro físico } \\
\text { y mental. } \\
\text { - Está basado en la sensación de pérdida. } \\
\text { - La depresión reactiva asociada a las consecuencias que tiene padecer una enfermedad } \\
\text { degenerativa, procedimientos médicos con efectos en la apariencia física. } \\
\text { - La depresión preparatoria está en afrontar los costos elevados de la enfermedad. }\end{array}$ \\
\hline 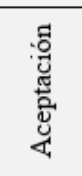 & $\begin{array}{l}\text { - Se despoja de los sentimientos transcurridos en el proceso de duelo. } \\
\text { - Evitan las perturbaciones del mundo real. } \\
\text { - Llegan a un estado de paz, aceptación y tranquilidad, en espera que pase lo inevitable. } \\
\text { - Percepción de que la muerte es ineludible, pero con la esperanza de vida en algunos } \\
\text { casos. }\end{array}$ \\
\hline
\end{tabular}

Nota: Elaboración propia a partir de las concepciones de Kübler-Ross (1993). 
Es fundamental que el agente pase por las etapas anteriores, donde se niegue a sí mismo su situación, despoje la ira que siente por estar en esa situación, trate de negociar y se sienta deprimido por lo que le está pasando; todo esto con el fin de llegar a la aceptación de la enfermedad que sufre y las consecuencias inevitables (J. Hernández, 2015; Kübler-Ross, 1993).

En la siguiente sección se presenta la problemática de duelo, articulada al proceso de formación de la estrategia, para buscar contribuir en la explicación sobre el comportamiento que tendría un agente ante la caída de una estrategia deliberada.

Duelo de la estrategia deliberada para el surgimiento de la estrategia emergente. Bowlby (1993) afirma que el uso restrictivo del concepto del duelo (solo para situaciones de muerte) va en contravía del pensamiento investigativo; no permite la evolución de la definición y encasillamiento de la visión del mundo. Se hace referencia a esta discusión para poder hacer uso del término de duelo, con el fin de denotar el proceso de ruptura que sufre un agente cuando es deconstruida la estrategia deliberada (J. Hernández, 2015; J. Hernández et al., 2015; Montoya y Montoya, 2013). La explicación de este proceso estará basada en las etapas descritas en la sección anterior, desarrolladas por Kübler-Ross (1993).

Cuando un agente genera una estrategia deliberada para lograr un objetivo, ejecuta diferentes fases del proceso de planeación respectivo y determina todas las etapas de un plan metódico para alcanzar la meta; calcula los costos previsibles relacionados a la ejecución y determina cuáles son las fuentes de financiación para lograr convertir la estrategia pretendida en realizada, generándose un vínculo entre el agente y la estrategia deliberada. Pero, por alguna razón relacionada con el ambiente o alguna consideración omitida por el agente en el proceso de planeación para la ejecución de la estrategia deliberada, existe la posibilidad de que esta se vea afectada y se convierta en inservible para llegar a la estrategia realizada (J. Hernández, 2015; J. Hernández et al., 2015; Montoya y Montoya, 2013).

La ruptura del vínculo del agente con la estrategia deliberada y la destrucción del enlace con el proceso de planeación y dedicación en la pretensión de la ejecución del mismo, de manera fiel, genera en el agente un proceso similar al de duelo (J. Hernández, 2015; J. Hernández et al., 2015). El agente va a sentir la pérdida, en donde la estrategia deliberada va a estar desenfocada de la realidad. La ruptura del vínculo y la sensación de pérdida, activan el proceso de duelo, ya que por definición es la respuesta normal a la pérdida (Bowlby, 1993).

Según Kübler-Ross (1993), la primera etapa del duelo es la negación. El agente, al momento de surgimiento de un obstáculo, el cual indica que su estrategia deliberada no va a poder ser aplicada, va a entrar en la etapa de negación, en donde cuestiona inicialmente la existencia del elemento truncador, lo ignora y busca la forma de continuar con su plan; el agente, al igual que los pacientes enfermos próximos a morir, utilizará la negación como una defensa al mundo real, buscando continuar con sus pretensiones. Adicionalmente, el agente en esta etapa de negación destaca la perfección de su estrategia deliberada y la imposibilidad de que falle, ya que él hace relucir sus habilidades como planeador y la rigurosidad con la que concibió todas las etapas de la situación para llegar a la estrategia realizada. De igual forma buscará otros conceptos frente al elemento obstaculizador, todas sus características de espacio y tiempo, y cómo afecta de manera directa e indirecta al plan deliberado (J. Hernández, 2015; J. Hernández et al., 2015).

Luego de pasar por la negación, donde no considera la posibilidad de fallo de su plan, continuaría una etapa similar a la ira, donde el agente empieza a reconocer que su plan está fracasando, busca los responsables de la situación que está obstaculizando su estrategia deliberada y descarga la irritación y el sentimiento de frustración que tiene por la pérdida de su apego al plan (J. Hernández, 2015; J. Hernández et al., 2015). 
Estas dos primeras etapas del duelo de la estrategia deliberada son agudizadas por el miedo que puede tener el agente a la aleatoriedad del futuro, ya que no sabe con certeza qué va a pasar, en instantes de tiempo posteriores, con el surgimiento del elemento truncador, que perturba de manera considerable la temporalidad y la armonía de las actividades dispuestas en el plan, hasta el punto de que se pueda convertir en obsoleto y se genere una total caída de la estrategia deliberada (J. Hernández, 2015; J. Hernández et al., 2015).

En ese instante, el agente quedará expuesto al ambiente sin ninguna estrategia actual o vigente con la cual enfrentarlo. Esto lo llevará a iniciar la etapa siguiente, de negociación, donde el agente buscará mediar la situación con su plan y de alguna forma darle continuidad y salvarlo, aunque tenga que hacer algunos cambios. En esta etapa el agente se cuestiona a sí mismo sobre qué puede hacer a partir del obstáculo presentado a su plan. De igual forma, se dará cuenta que su estrategia deliberada ha fracasado y comenzará a considerar nuevas alternativas que le hagan llevadera la situación; el agente busca en sus experiencias pasadas alternativas que funcionen para cumplir el objetivo, o que por lo menos haga más tolerable la situación (J. Hernández, 2015; J. Hernández et al., 2015).

La siguiente etapa es similar a la de depresión, contextualizada en dos pasos; el agente eventualmente se siente triste por su inminente pérdida, inicia el reconocimiento de que su plan va a fallar en la consecución del objetivo, lo cual puede estar enmarcado inicialmente en una depresión reactiva. Posteriormente, la depresión preparatoria, está relacionada con la disposición de recursos para que el agente continúe siendo viable en el sistema para tomar decisiones (J. Hernández, 2015; J. Hernández et al., 2015).

Finalmente, la última etapa es la aceptación, donde definitivamente el agente acepta que su estrategia deliberada no funcionó y que es inviable para llegar a ser una estrategia realizada. En esta etapa el agente deja de lado los sentimientos que tiene por su plan. En la tabla 2 se pueden observar las características de cada una de las etapas.

\section{Tabla 2}

Etapas del duelo de la estrategia deliberada

\begin{tabular}{|c|c|}
\hline Etapa & Características \\
\hline Negación & $\begin{array}{l}\text { - El agente se defiende de la realidad. } \\
\text { - Destaca su habilidad como planeador. } \\
\text { - Cuestiona la imposibilidad de fallo de su plan. }\end{array}$ \\
\hline Ira & $\begin{array}{l}\text { - Aceptación parcial de un elemento truncador que afecta su estrategia } \\
\text { deliberada. } \\
\text { - La ira proviene de la ruptura del plan. } \\
\text { - Descarga su ira contra lo que lo rodea. }\end{array}$ \\
\hline Negociación & $\begin{array}{l}\text { - Se cuestiona alternativas preliminares para poder continuar con el plan. } \\
\text { - Realiza consideraciones con respecto a la continuidad del plan. } \\
\text { - Consulta en experiencias pasadas para buscar una solución. }\end{array}$ \\
\hline Depresión & $\begin{array}{l}\text { - El agente siente tristeza por la pérdida del plan. } \\
\text { - Deja de tener una actitud positiva ante el plan. }\end{array}$ \\
\hline Aceptación & $\begin{array}{l}\text { - Deja de lado los sentimientos. } \\
\text { - Acepta definitivamente la muerte de la estrategia deliberada. } \\
\text { - Es necesario pasar por las otras etapas para poder llegar a acepta la ruptura del } \\
\text { vínculo entre la estrategia deliberada v el agente }\end{array}$ \\
\hline
\end{tabular}

Nota: tomado de J. Hernández et al., 2015, p. 554. 
Si un agente no hace el proceso de duelo de su estrategia deliberada, probablemente, por orgullo o miedo, fracasará en la consecución del objetivo, pero con certeza retrasará la posibilidad de tomar decisiones, al punto de que ya no se mantenga viable en el sistema para continuar tomando decisiones (J. Hernández, 2015; J. Hernández et al., 2015).

Otros agentes pueden pasar por algunas etapas, pero sin llegar a la aceptación. El agente puede quedarse en la etapa de negación y persistir en su estrategia deliberada sin reconocer su inviabilidad. De igual forma, en la etapa de ira, si el agente se queda en esa etapa, reprochando al ambiente y a sí mismo el fracaso de su plan, no va a poder continuar el camino hacia el objetivo. También, llegar a un estado de conciencia que le permite entender que está fracasando, pero no llegar a un acuerdo en el proceso de negociación entre su plan y el ambiente. A su vez puede pasar que se bloquee, porque se quedó lamentando el fracaso de su plan. Si el agente no llega a la aceptación del fracaso del plan, no podrá abrir la puerta al surgimiento de una estrategia emergente que le permita continuar viable en el sistema (J. Hernández, 2015; J. Hernández et al., 2015).

\section{| Materiales y métodos}

En el apartado anterior, se desarrolló una relación teórica entre el proceso de duelo y el proceso de formación de la estrategia, donde se mostró cómo el agente pasa por las etapas de duelo al momento de tener una ruptura del vínculo con las estrategias deliberadas, y cómo se presume el surgimiento de la estrategia emergente cuando supere las etapas. En esta sección se describe el uso del "juego de la cerveza" como prueba para identificar la existencia de las etapas del proceso de duelo cuando ocurre una deconstrucción de una estrategia deliberada y posterior ruptura de la relación entre el agente y el plan, propuesto en J. Hernández et al. (2015).

El juego de la cerveza es una actividad didáctica desarrollada en los años 60 por el Instituto Tecnológico de Massachusetts - MIT, en la cual se permite visualizar la dinámica de un sistema de producción y distribución de una cervecería; cada uno de los jugadores está en la libertad de tomar decisiones y pretender maximizar sus ganancias. El juego cuenta con los siguientes roles en la cadena de distribución: minorista, mayorista y productor; cada uno realiza una operación de compra al siguiente rol (J. Hernández et al., 2018; Senge, 1995). Principalmente se ha usado como una herramienta didáctica para explicar el efecto látigo de la cadena de abastecimiento; también, con algunos ajustes se pueden ver los efectos de mejoras en la comunicación o en tiempos de reabastecimiento (Roser et al., 2020) y la teoría de inventarios (Alfieri \& Zotteri, 2016).

Puntualmente, para esta investigación, se tomó el juego de la cerveza como herramienta para crear un escenario controlable, en donde los participantes pudieran generar planes y tomar decisiones. Específicamente, se esperaba que cada uno de los jugadores, representando uno de los roles, formara una estrategia a partir del patrón en el flujo de decisiones planeadas que estaba generando con cada uno de los pedidos, lo que convierte a esa estrategia en una estrategia deliberada. Para dar el efecto de deconstrucción de la estrategia que fue planeada, se generó la terminación abrupta del juego de la cerveza, lo que en consecuencia permitió evaluar la sensación que tiene el agente en la ruptura del vínculo con la estrategia deliberada (J. Hernández, 2015).

Detalladamente, la metodología usada dentro de este documento tiene las siguientes fases (J. Hernández, 2015): 
Fase 1: Indicación de instrucciones. Se dispusieron a los participantes en una sala de computo, cada uno con un computador, que cuenta con el software "The beer game portal", aplicación virtual del juego de la cerveza (Riemer \& Christ, 2012). El moderador explicó la dinámica, indicando que cada uno de los participantes tenía un rol dentro de la cadena de suministro (minorista, mayorista y fabricante), y se esperaba que tomaran las decisiones sobre la cantidad a ordenar a otro miembro de la cadena de suministro; teniendo en cuenta los costos de dejar de atender demanda, los costos de inventario y el periodo que tarda el abastecimiento. Se les indicó a los participantes que había un premio para el que realizara el mejor manejo de los costos, a manera de incentivo.

Fase 2: Aplicación del juego. Se ejecutó el juego y los participantes empezaron a realizar órdenes a los demás miembros de la cadena de suministro, esperando mantener sus costos bajos. El moderador anunció que el juego en su totalidad iba a constar de 50 semanas (es decir 50 decisiones por cada uno de los participantes). En las primeras semanas el moderador indicó a los participantes qué cantidad ordenar, con el fin de que se fueran familiarizando con la aplicación. A partir de la semana nueve, los participantes empezaron a tomar de manera individual la decisión de cuánta cantidad ordenar. Súbitamente y prematuramente en la semana 20 el juego fue finalizado, con el fin de generar deconstrucción de los planes de los participantes.

Fase 3: Aplicación de la encuesta. Al finalizar el juego de manera abrupta, a los participantes se les pidió diligenciar una encuesta, en la cual se tenía como objetivo identificar el impacto que tiene la ruptura de un plan y establecer las emociones que siente el agente en este proceso. En la tabla 3 se pueden evidenciar las variables que se tuvieron en cuenta en el diseño de la encuesta.

\section{Tabla 3}

Variables de la encuesta

\begin{tabular}{|c|c|c|}
\hline $\begin{array}{c}\text { Objetivo de } \\
\text { investigación }\end{array}$ & Variable & Descripción \\
\hline \multirow{3}{*}{ 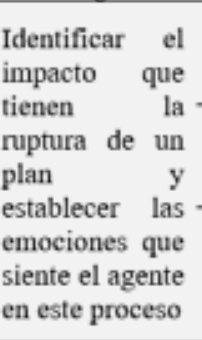 } & Vine & $\begin{array}{l}\text { Identificar la percepción } \\
\text { en el juego de la cerveza } \\
\text { cuando elaboran un plan. }\end{array}$ \\
\hline & $\begin{array}{l}\text { to } \\
\text { or que }\end{array}$ & $\begin{array}{l}\text { Identificar la percepción que tiene el agente asociado a la } \\
\text { finalización súbita del juego de la cerveza como elemento } \\
\text { truncador que deconstruye el plan. }\end{array}$ \\
\hline & $\begin{array}{l}\text { Etapas } \\
\text { proceso } \\
\text { duelo }\end{array}$ & $\begin{array}{l}\text { Identificar la valoración que le da el agenten a las etapas del } \\
\text { proceso de duelo (negación, ira, negociación, depresión y } \\
\text { aceptación) al momento de la deconstrucción de la estrategia } \\
\text { deliberada. }\end{array}$ \\
\hline
\end{tabular}

Nota: tomado de J. Hernández et al., 2015, p. 556. 
Con tal fin, se desarrolló el taller con los estudiantes de la asignatura Gestión Estratégica, de la Facultad de Minas de la Universidad Nacional de Colombia, soportado en la aplicación digital "The Beergame Software" (Riemer \& Christ, 2012). Se contó con la participación de 48 estudiantes, principalmente del programa de Ingeniería Industrial e Ingeniería Administrativa.

\section{Resultados}

Luego de la aplicación del juego de la cerveza y la finalización abrupta, con el fin de generar un suceso deconstructor que rompa el vínculo entre el agente y la estrategia deliberada, se realizó una encuesta, para identificar las sensaciones de los agentes en relación con el vínculo y las etapas del proceso de duelo.

Cuando se les preguntó a los participantes del taller sobre la sensación de vínculo al momento de diseñar un plan; es decir, qué tan vinculados se sienten a la estrategia deliberada, el $97,9 \%$ de los participantes informó que siente un vínculo con el plan, tan solo el 2,1 \% de los encuestados declaró que no siente vínculo con los planes.

Seguidamente, se les preguntó sobre los elementos que hacen que se genere ese vínculo entre el agente y el plan, donde sobresalen los elementos de tiempo, conocimiento y oportunidades; en una medida alta o muy alta el $91,7 \%$ de los participantes relacionó al tiempo con el vínculo, el $84,5 \%$ con el conocimiento y el $84,5 \%$ con las oportunidades. En la tabla 4 se pueden observar los resultados completos.

\section{Tabla 4}

Elementos asociados al vinculo con el plan

\begin{tabular}{|c|c|c|c|c|c|c|c|}
\hline \multirow[b]{2}{*}{$\begin{array}{l}\text { Elementos con los que } \\
\text { relaciona el vinculo con el plan }\end{array}$} & \multicolumn{7}{|c|}{ Percepción } \\
\hline & $\begin{array}{l}\text { Muy } \\
\text { alta }\end{array}$ & Alta & Media & Baja & $\begin{array}{l}\text { Muy } \\
\text { baja }\end{array}$ & Nulo & $\begin{array}{l}\text { No } \\
\text { sabe/no } \\
\text { responde }\end{array}$ \\
\hline Tiempo & $37.5 \%$ & $54.2 \%$ & $8.3 \%$ & $0 \%$ & $0 \%$ & $0 \%$ & $0 \%$ \\
\hline Conocimiento & $43.8 \%$ & $41.7 \%$ & $12.5 \%$ & $2.1 \%$ & $0 \%$ & $0 \%$ & $0 \%$ \\
\hline Oportunidad & $31,3 \%$ & $54,2 \%$ & $12,5 \%$ & $2,1 \%$ & $0 \%$ & $0 \%$ & $0 \%$ \\
\hline
\end{tabular}

\section{Nota: Elaboración propia con datos de J. Hernández (2015).}

Además, se evidencia que el 75 \% de los participantes informaron sensación de deconstrucción del plan, cuando se les preguntó sobre la finalización abrupta del juego y la inoperancia del plan en esas condiciones. Desde una consideración media, alta o muy alta, el 75 \% de los participantes declaró que no están de acuerdo con la finalización abrupta del juego, lo cual esboza elementos de negación. A su vez, el 56,3 \% de los participantes informó que, en un nivel medio, alto o muy alto sintió rabia por la deconstrucción del plan. Por otro lado, el 85,4\% expresó que quiere negociar por lo menos en un nivel medio. El 81,3 \% dijo que siente tristeza. Finalmente, desde un nivel medio, el 54,2 \% de los participantes demostró aceptación a la finalización abrupta del juego de la cerveza. En la tabla 5 se pueden observar los resultados completos, y en la figura 1 un resumen de los resultados. 
Tabla 5

Etapa del proceso de duelo ante el hecho del elemento truncador

\begin{tabular}{lccccccc}
\hline & \multicolumn{7}{c}{ Percepción } \\
\cline { 2 - 8 } Elementos del proceso de duelo & $\begin{array}{l}\text { Muy } \\
\text { alta }\end{array}$ & Alta & Media & Baja & $\begin{array}{l}\text { Muy } \\
\text { baja }\end{array}$ & Nulo $\begin{array}{l}\text { No } \\
\text { sabe/no } \\
\text { responde }\end{array}$ \\
\hline Negación & $18,8 \%$ & $29,2 \%$ & $27,1 \%$ & $14,6 \%$ & $6,3 \%$ & $2,1 \%$ & $2,1 \%$ \\
\hline Ira & $6,3 \%$ & $22,9 \%$ & $27.1 \%$ & $22,9 \%$ & $10,4 \%$ & $8,3 \%$ & $2,1 \%$ \\
\hline Negociación & $29,2 \%$ & $33,3 \%$ & $22,9 \%$ & $8,3 \%$ & $2,1 \%$ & $2,1 \%$ & $2,1 \%$ \\
\hline Tristeza & $29,2 \%$ & $20,8 \%$ & $31,3 \%$ & $6,3 \%$ & $8,3 \%$ & $2,1 \%$ & $2,1 \%$ \\
\hline Aceptación & $6,3 \%$ & $16,7 \%$ & $20,8 \%$ & $29,2 \%$ & $22,9 \%$ & $2,1 \%$ & $2,1 \%$ \\
\hline
\end{tabular}

Nota: Elaboración propia con dados de J. Hernández (2015).

\section{Figura 1}

Declaraciones ante eventos deconstructores

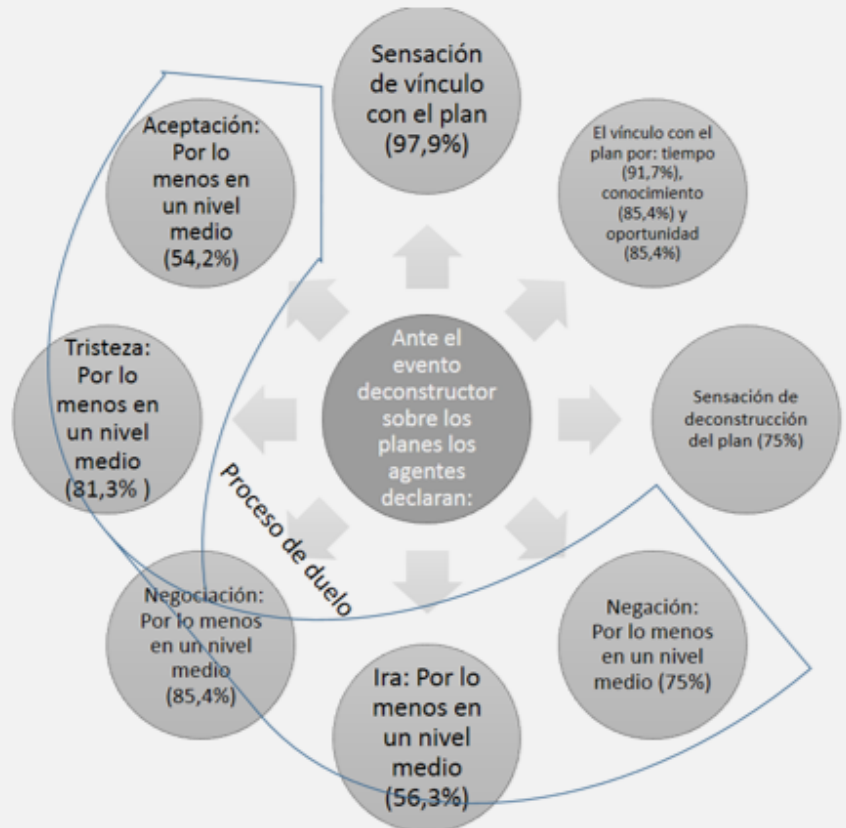

Nota: Elaboración propia. 


\section{Discusión}

A partir de los resultados obtenidos, en el apartado anterior, se puede ratificar, de manera preliminar, la posibilidad del uso del concepto de duelo dentro del proceso de formación de la estrategia; ya que Bowlby (1993) pretende que este concepto pueda ser extrapolado a otras perspectivas diferentes a la pérdida por la muerte de un ser querido. De hecho, al indagar a los participantes del estudio, manifestaron de manera contundente su relación vinculante con el plan; esto puede llevar a pensar que la ruptura con dicho plan puede generar reacciones iguales o similares al proceso de duelo en el agente.

Se puede decir que los participantes sufrieron una ruptura del plan, dado que manifestaron su inconformidad por la finalización abrupta del juego, y en consecuencia de su plan. También, se pudo indagar que ese vínculo entre el agente y el plan está justificado a partir de los elementos de tiempo, conocimiento y oportunidades, que representan el esfuerzo dedicado por parte del agente en la elaboración y desarrollo de la estrategia que fue truncada.

Con respecto a las aportaciones de Kübler-Ross (1993), referente a las etapas de duelo, el sentimiento que más floreció dentro de los participante fue la pretensión de querer negociar la situación, lo que da a entender la aspiración de seguir estando viable dentro de la situación, con el fin de poder continuar tomando decisiones (J. Hernández, 2015; J. Hernández et al., 2015; J. Hernández et al., 2019). El siguiente sentimiento más común fue la tristeza, relacionada con la depresión del agente, ante el reconocimiento de la pérdida de su plan, pero también se puede convertir en el motor de la oportunidad para construir una nueva estrategia (estrategia emergente). Luego, aparece la negación con un firme deseo de seguir el juego, sin reconocer la realidad de la finalización abrupta o inesperada. Los sentimientos del proceso de duelo menos masivos fueron la ira y la aceptación; no obstante, a pesar de esto, más de la mitad de los participantes manifestaron sentir, por lo menos, de una manera media estos sentimientos. Lo que si queda claro es que el proceso de duelo participa como una etapa de transición; podría decirse que entre la estrategia deliberada y emergente, en donde, en términos de Cognet \& Masson (2019), corresponde a sublimación y resurgimiento del individuo.

Otro elemento a destacar es como se relaciona la incertidumbre en los procesos de duelo con las deconstrucciones de estrategias deliberadas y surgimientos de estrategias emergentes. La incertidumbre, en el contexto del duelo psicológico, surge por las manifestaciones de preocupación relacionada con vínculos significativos por parte de la persona que está pasando por este proceso, sobretodo asociados a asuntos que están bajo su responsabilidad y el sinsabor de no poder continuar enfrentando ese compromiso (Díaz et al., 2013). Según Gómez et al., (2016), la incertidumbre se destaca en los procesos de duelo porque la percepción de futuro está fuertemente relacionada con la ansiedad y angustia de no existencia en un instante más allá del actual; lo que lleva a una revaluación y renovación de las acciones ejecutadas en el presente que otorgue en cierta medida una esperanza de vida. En el contexto de la deconstrucción de las estrategias deliberadas, los agentes deben realizar una revaluación de los planes y renovaciones de las acciones y decisiones, que les permitan generar estrategias emergentes que les garantice viabilidad en el futuro para alcanzar los objetivos.

En resumen, los resultados expuestos en el apartado anterior permiten identificar, como valor agregado de esta investigación, que en efecto existe un vínculo del agente con el plan, que está asociado a elementos de tiempo, oportunidades y conocimiento, y que cuando se genera la deconstrucción del mismo, el agente expresa sentimientos que están asociados al proceso del duelo (Bowlby, 1993; J. Hernández, 2015; J. Hernández et al., 2015; Kübler-Ross, 1993). Hará parte de investigaciones futuras determinar la intensidad de esas sensaciones dentro de ese proceso de duelo e identificar cómo se desarrolla en situaciones estratégicas dentro del mundo organizacional. 
Finalmente, a manera de implicaciones prácticas, los resultados permiten sumar los elementos del proceso de duelo psicológico, como sensaciones o etapas que debe pasar un agente tomador de decisiones ante la inoperancia efectiva de su plan; en donde sí son superadas efectivamente, permitirá eventualmente el surgimiento de la estrategia emergente, la cual le consentirá continuar siendo viable dentro del sistema en el cual se está desenvolviendo y podrá seguir tomando decisiones que le permiten construir su estrategia, sobre todo en entornos cambiantes.

\section{| Conclusiones}

El proceso de duelo se va a hacer presente ante disolución de la relación entre el agente y su plan. Dicha situación se manifiesta dentro del proceso de formación de la estrategia, cuando la estrategia deliberada es deconstruida por un elemento truncador, donde se cree conveniente que el agente inicie el proceso de duelo psicológico.

En consecuencia con lo anterior, para que florezca la posibilidad del surgimiento de la estrategia emergente, el agente debe acaecer por las etapas del proceso de duelo de la estrategia deliberada frustrada; no será simple reconocer el fracaso de dicha estrategia y posterior búsqueda de alternativas emergentes, si el agente no alcanza la aceptación (última etapa del proceso de duelo) de la deconstrucción del plan.

Estos dos factores posiblemente serían la razón por la cual el agente no se encaminaría en el proceso de duelo de la estrategia deliberada. El primer factor estaría establecido en el marco del orgullo, que acompaña al actor, ya que se encuentra obnubilado por el esfuerzo y dedicación de tiempo en el desarrollo del plan; resaltando su perfección e infalibilidad y tratando de rescatar los recursos invertidos, a pesar de que ya la factibilidad haya desaparecido. El segundo, se encuentra en el marco del miedo; para el agente es innegable que su plan ha fracasado, pero su ignorancia frente al futuro o la falta de herramientas para afrontarlo, lo llevan a aferrarse de manera considerable a su estrategia deliberada, a pesar de su inviabilidad.

Del resultado de la aplicación del taller se puede concluir que el agente genera vínculo con el plan, es decir con las estrategias deliberadas; además, cuando surge un elemento truncador que la deconstruye, se genera una ruptura del vínculo, en el cual el agente percibe las sensaciones, características del proceso de duelo psicológico.

\section{Referencias}

Alfieri, A., \& Zotteri, G. (2016). Inventory theory and the Beer Game. International Journal of Logistics Research and Applications, 20(4), 381-404. https://doi.or-

g/10.1080/13675567.2016.1243657

Americam Psychiatric Association. (2014). DSM-5 (5 ed.). Americam Psychiatric Association.

Díaz, V., Ruiz, M., Flórez, C., Urrea, Y., Córdoba, V., Arbeláez, C. y Rodríguez, D. (2013). El proceso de morir: destino y significación del diagnóstico de muerte inminente. Revista Virtual Universidad Católica del Norte, (39), 195-211. https://revistavirtual.ucn.edu.co/index.php/RevistaUC$\mathrm{N} /$ article/view/436/892

Bowlby, J. (1993). La perdida afectiva. Paidós Ibérica.

Cognet, A., \& Masson, C. (2019). Remember. Mourning and the creative process. L'evolution Psychiatrique, 84, 1-9. https://doi.org/10.1016/j.evopsy.2019.07.007

Feigelman, W., \& Cerel, J. (2020). Feelings of blameworthiness and their associations with the grieving process in suicide mourning. Frontiers in Psychology, 11, 1-9. https://doi.org/10.3389/fpsyg.2020.00610 
Fonseca, M. (2010). El duelo neonatal y su manejo. Asociación Mexicana de Educación Continua y a Distancia.

Ghezzi, A. (2013). Revisiting business strategy under discontinuity. Management Decision, 51(7), 1326-1358. https://doi.org/10.1108/MD-05-2012-0388

Gómez Osorio, Á. M., Castañeda Ruiz, H. N., Sánchez Sánchez, L. F. y Lotero Castañeda, J. C. (2016). Un acercamiento a los dilemas bioéticos de la enfermedad y la muerte a partir de un ejercicio de análisis cinematográfico. Revista Virtual Universidad Católica del Norte, (49), 183-205. http://revistavirtual.ucn.edu.co/index.php/RevistaUCN/article/view/804/1324

Hax, A., \& Majluf, N. (1988). The concept of strategy and the strategy formation process. Interfaces, 18(3), 99-109. https://doi.org/10.1287/inte.18.3.99

Hernández, A. (2006). La decisión y su relación con el tiempo: Estrategia, procesos e identidad. Revista Facultad de Ciencias Económicas: Investigación y Reflexión, 14(1), 23-43.

https: // www.redalyc.org/articulo.oa?id=90900104

Hernández, J. (2015). Contribución a la comprensión del momento de la decisión estratégica Formulación de un taller de estrategia orientado al momento de la decisión. Universidad Nacional de Colombia.

Hernández, J., Montoya, I., \& Montoya, L. (2015). The mourning of the deliberate strategy for the arising of the emergent strategy. Tojet, (1), 547-556. http://www.tojet.net/special/2015_7_1.pdf

Hernández, J., Montoya, I., \& Montoya, L. (2017). The moment of decision from the perspective of deliberate and emergent strategies. Ad-Minister, 31, 93-114. https://doi.org/10.17230/ad-minister.31.6

Hernández, J., Montoya, I., \& Montoya, L. (2018). Strategic decision-moment: Beer game comparison between two colombian universities. The International Journal of Management Education, 16(3), 504-514. https://doi.org/10.1016/j.ijme.2018.10.001

Hernández, J., Montoya, L., \& Montoya, I. (2019). Deliberate strategy deconstructing event for the arising of the emergent strategy. Journal of Engineering and Applied Sciences, 11(22), 8452-8463. https://doi.org/10.36478/jeasci.2019.8452.8463

Kariman, N., Simbar, M., Ahmadi, F., \& Vedadhir, A. A. (2014). Socioeconomic and emotional predictors of decision making for yiming motherhood in iranian women in 2013. Iranian Red Crescent Medical Journal, 16(2), 1-8. https://doi.org/10.5812/ircmj.13629

Kübler-Ross, E. (1993). Sobre la muerte y los moribundos (4 ed.). Grijalbo.

Londoño-Patiño, J. A., y Acevedo-Álvarez, C. A. (2018). El aprendizaje organizacional (AO) y el desempeño empresarial bajo el enfoque de las capacidades dinámicas de aprendizaje. Revista CEA, 4(7), 103-118. https://doi.org/10.22430/24223182.762

MacLean, D., \& MacIntosh, R. (2015). Planning reconsidered: Paradox, poetry and people at the edge of strategy. European Management Journal, 33(2), 72-78. https://doi.or-

g/10.1016/j.emj.2015.02.003

March, J., \& Simon, H. (1958). Organizations. Wiley y Sons.

Mintzberg, H. (1978). Patterns in strategy formation. Management Science, 24(9), 934-948. https://doi.org/10.1080/00208825.1979.11656272

Mintzberg, H. (1987). The strategy concept I: Five Ps for strategy. California Management Review, 11-24. https://doi.org/10.2307/41165263

Mintzberg, H., \& Jorgensen, J. (1987). Emergent strategy for public policy. Canadian Public Administration, 30(2), 214-229. https://doi.org/10.1017/CBO9781107415324.004

Mintzberg, H., \& McHugh, A. (1985). Strategy formation in an adhocracy. Administrative Science Quarterly, 30(2), 160-197. https://doi.org/10.2307/2393104

Mintzberg, H., \& Waters, J. (1985). Of strategies, debilberate and emergent. Strategic Management Journal, 6(3), 257-272. https://doi.org/10.2307/2486186

Montoya, I. (2010). Una contribución a la comprensión de las estrategias deliberadas y emergentes de las organizaciones, desde una perspectiva evolutiva. Universidad Nacional de Colombia. 
Montoya, I., y Montoya, L. (2009). Algunas concepciones del tiempo en ciencias sociales e implicaciones para la investigación en dirección estrategica. Revista Facultad de Ciencias Económicas de La Universidad Militar de Nueva Granada, 17, 57-76. https://doi.org/10.18359/rfce. 4453

Montoya, I., y Montoya, L. (2013). La formación de estrategias deliberadas y emergentes: Una propuesta a partir de definiciones básicas de una metología de sistemas suaves. Revista Facultad de Ciencias Económicas: Investigación y Reflexión, 21(2), 67-96. https://doi.org/10.18359/rfce.657

Paulus, M. (2005). Neurobiology of decision-making: Quo vadis? Cognitive Brain Research, 23, 2-10. https://doi.org/10.1016/j.cogbrainres.2005.01.001

Quinn, J. (1978). Strategic change: "Logical Incrementalism”. Sloan Management Review, 20(1), 7-21. https://doi.org/10.5465/amr.1982.4285636

Real Academia Española. (2014). Diccionario de la lengua española: Duelo. https://dle.rae.es/duelo?m=form

Riemer, K., \& Christ, F. (2012). The Beergame software [Software]. https://beergame.org/

Roser, C., Sato, M., \& Nakano, M. (2020). Would you like some wine? Introducing variants to the beer game. Production Planning and Control, 1-9. https://doi.org/10.1080/09537287.2020.1742370

Saavedra, J. (2006). El tiempo y la decisión en la organización desde una perspectiva cultural. Revista Facultad de Ciencias Económicas Investigación y Reflexión, 14(1), 44-65. https://www.redalyc.org/articulo.oa?id=90900105

Sánchez, C. (2020). Del duelo normal al duelo patológico: Abordaje psicológico (2 ed.). Punto Rojo Libros S.L. https:// books.google.com.co/books?id=(ZXLDWAAQBAJ

Senge, P. (1995). La quinta disciplina. Verdana.

Simon, H. (1955). A Behavioral Model of Rational Choice. The Quarterly Journal of Economics, 69(1), 99-118. https://doi.org/10.2307/1884852

Zaragoza, R. (2007). Estilos de afrontamiento ante una separación amorosa en personas con pérdida parental en la infancia. Universidad de las Américas Puebla. 